\title{
Linguistik als Kampfsport - Ein Plädoyer für die Suche nach Pa- radigmen demokratischen Sprechens in Alltag, Medien und Recht
}

\author{
Friedemann Vogel (Freiburg i. Br.)
}

\begin{abstract}
Following Bourdieu's notion of applied research this contribution asks for the possibility of linguistics as a kind of "martial art" and "self-defence", i. e. linguistics trying to interfere in society based on a holistic model of human rights and paradigms of democratic speech. In this perspective the text discusses elements of a normative model, which is able to lead linguistic research practices and explores the context of education, law and the media formation of participation. Finally I will consider the meaning of speech practices and linguistic research or methods in the context of capitalism and profit.
\end{abstract}

\section{$1 \quad$ Einführung}

Pierre Bourdieu prägte in einem Radiointerview einmal den Satz, "Soziologie [sei] ein Kampfsport" (Carles/Bourdieu 2009). Er meinte damit, dass Analyse und Verständnis des sozialen Handelns dazu beitragen können, gesellschaftliche Herrschaftsverhältnisse zu durchschauen und zu verändern. ${ }^{1}$ Das Verständnis müsse in eine soziologische Theorie der Praxis überführt und als solche selbst zur gesellschaftlichen Praxis, zur gesellschaftlichen Intervention werden:

Die Theorie der Praxis als Praxis ist das einzige Mittel, um der Alternative zwischen Materialismus und Idealismus zu entgehen, indem sie dem positivistischen Materialismus entgegenhält, daß ihre Gegenstände konstruiert sind, dem intellektualistischen Idealismus dagegen, daß das Prinzip dieser Konstruktion die praktische, auf praktische Funktionen ausgerichtete Tätigkeit ist.

(Bourdieu 2009: 412, Fn. 17)

Bourdieu war ein Analytiker und er war Kritiker, der in der Öffentlichkeit nicht nur mit seinen Büchern, sondern zum Beispiel auch auf Demonstrationen die Schlussfolgerungen aus seinen Studien lebte.

Bourdieus Arbeit hat mich immer sehr beeindruckt und in mir die Frage bewegt, wie denn eine "Linguistik als Kampfsport" auszusehen hätte? Was können Sprach- als Kulturwissenschaftler dazu beitragen, das soziale Zusammenleben in unserer Gesellschaft zu verbessern? Welche Antworten oder zumindest Orientierungsrahmen können wir anbieten auf die Frage, wie unsere Welt aus linguistischer Perspektive einzurichten wäre und also aus Menschen "mündige" Bürgerinnen und Bürger werden?

\footnotetext{
${ }^{1}$ Sein Schüler Wacquant hat das nochmal anders aufgegriffen und ist für seine Studien in amerikanischen Ghettos für drei Jahre zum Boxer geworden.
}

Linguistik online 69, 7/14 - http://dx.doi.org/10.13092/lo.69.1658

licensed under CC 3.0 
Derartige Fragen sind natürlich nicht neu - denken wir allein nur an die Arbeiten der Sprachkritik, an die Initiative der Unwörter des Jahres, an die Arbeiten zu Mehrsprachigkeit und Migration, zu Schlichtungsgesprächen und vielem anderen. Allerdings tun sich Sprachwissenschaftler in der Regel eher schwer mit normativen respektive kritisierenden Aussagen. Das hat wohl verschiedene Gründe. Ein wichtiger Grund dürfte schon der pragmatische Zuschnitt zeitgenössischer Linguistik und ein daraus resultierender deskriptiver Selbstanspruch sein. Sprache ist demnach mit Wittgenstein (1953/2003) regelhaft (konventionalisiert), aber eben nicht normativ (bestenfalls kann regelhaftes Verhalten für Beobachter Orientierungs- und Anpassungsprozesse aktivieren). Ein sprachlicher Ausdruck selbst ist nicht richtig oder falsch, er kann nur situativ angemessen oder eben unangemessen gebraucht werden. Sprachwissenschaftler bzw. linguistische Sprachkritiker wollen seit Wimmer (1982/1988) daher wenn, dann nur multiperspektivisch Sprachgebrauch beschreiben und dem einzelnen Akteur die jeweiligen Folgen seines Sprachgebrauchs reflektierend vor Augen führen. Kritik im Kant'schen Sinne. Die normative Entscheidung bleibt bei dem Akteur selbst. Mit Bezug auf Mehrsprachigkeitsverhältnisse und Sprachenpolitik skizziert Coulmas (2009: 178) das Problem ganz treffend:

most linguists would say that it is absurd to talk about languages changing for the better or the worse. They chance, and that is all. There are two versions of this point of view. One is that language change can be influenced as much as the terrestrial climate. There is really no point in trying to do it. The other is that the very attempt to exercise an influence on language is unscientific and hence not what linguists are being paid for. Whether we can or cannot influence language change is unimportant; we should not do it. The metaphor of language as an organism has influenced the notion of what a language is so strongly that any attempt at influencing its natural development is morally as dubious as cloning. Most linguists have gladly adopted either the 'cannot' or the 'should not' position, and by declaring themselves incompetent in matters of 'good' or 'bad' language have relieved themselves of responsibility for the possible consequences of their own work.

Damit wird auch noch ein anderer Hintergrund angedeutet: normativen Aussagen durch Wissenschaftler haftet heute generell der Stallgeruch des Instrumentell-Politischen, gar Parteipolitisch-Ideologischen an - in der Linguistik vielleicht sogar mehr als in anderen Fachbereichen. ${ }^{2}$ Wissenschaft aber, so die vorherrschende Vorstellung schon seit Max Weber, habe im ideologischen Sinne apolitisch zu sein. Normativ-politische Aussagen sind etwas für Juristen, Politiker oder für den Stammtisch; "Politik gehört nicht in den Hörsaal" (Weber 1919/2002: 496497).

Ich möchte auf den folgenden Seiten dennoch überlegen, zunächst, ob es einen allgemeinen Orientierungsrahmen für normative, linguistische Aussagen geben kann, eine Art Kompass zur sprachwissenschaftlichen Bewertung gesellschaftlicher Verhältnisse. Ausgehend von einem holistischen Demokratie- und Menschenrechtskonzept (vgl. dazu schon Vogel 2012a: 286-287) werde ich anschließend kurz an zwei, meines Erachtens grundlegendenden Gesellschaftsbereichen skizzenhaft der Frage nachgehen, welche Möglichkeitsbedingungen für ein - wie ich es salopp nennen möchte - demokratisches Sprechen gegeben sein müssten, ehe ich dann im Fazit noch einmal zur "Linguistik als Kampfsport" zurückkomme.

\footnotetext{
${ }^{2}$ Im Mainstream aktueller Politikwissenschaft findet sich dagegen umkehrt vielfach unmittelbare Politik im Kleide "objektiver" Zahlenspiele.
} 


\section{$2 \quad$ Ein Kompass für Linguisten?}

Kann es eine Art Kompass für Linguisten, einen allgemeinen Wertmaßstab für sprachwissenschaftliche normative Aussagen, für linguistisch wertende Kritik geben? - Diese Frage rührt zunächst an eine viel grundlegendere Frage, nämlich: Was ist Sinn und Zweck linguistischer Wissenschaft überhaupt? Linke et al. (2004) schreiben dazu etwa in ihrer vielgenutzten Einführung in die Linguistik:

Sprachwissenschaft [oder syn.: Linguistik, FV] ist die Wissenschaft von der Sprache. [...] Ziel der Sprachwissenschaft ist die Beschreibung und Erklärung sprachlicher Phänomene, und sie tut dies in vorwiegend theoretischer Absicht. [...] Als theoretische Wissenschaft besitzt die Linguistik im Unterschied zu anwendungsorientierten Wissenschaften aber kein unmittelbar zugeordnetes Praxisfeld. Wer gut schreiben lernen will oder Sprachlehrer wird oder wissen möchte, wie man eine gute Rede hält, mag aus der Sprachwissenschaft viele nützliche Dinge lernen. Er findet für seine Praxisziele aber keine direkten Anleitungen.

(Linke et al.: 2004: 1; Unterstreichungen durch F. V.)

Ziel sei also vornehmlich die Beschreibung und Erklärung, nicht die Bewertung sprachlichkommunikativer Praxis. Dieses Grundverständnis wird kontrastiert oder zumindest erweitert durch Sprachwissenschaftler, die nach Wegen einer linguistischen Aufklärung suchen. Feilke, Knobloch und Völzing (2007) schreiben hierzu etwa:

Den Anspruch, zur 'Aufklärung' beizutragen, kann linguistisches Spezial- und Expertenwissen nur dann geltend machen, wenn es zugleich mit dem Nutzen, den es für die Allgemeinheit stiftet, auch zur Herstellung 'vernünftiger' sprachlich-kommunikativer Verhältnisse beiträgt. Den (durchaus nicht pathosfreien) Titel der 'Aufklärung' wollen wir fachlichen Aktivitäten sicher nur dann zubilligen, wenn das wohl verstandene, langfristige und universalistische Bedürfnis der Allgemeinheit nach vernünftigen Verhältnissen durch sie befördert wird und wenn sie den einzelnen dazu befähigen, aus undurchschauten Macht- und Abhängigkeitsbeziehungen herauszutreten.

(Feilke/Knobloch/Völzing 2007: 12)

In diesem Auszug wird von Sprachwissenschaftlern implizit auch erwartet, zu sprachlich-kommunikativen Phänomenen in der Gesellschaft Stellung zu beziehen, ergo zu bewerten. Aufklärung wird hier verstanden als aktive Aufgabe der Linguistik, für die Allgemeinheit gesellschaftliche Handlungsoptionen zu entwickeln. Nicht nur "kritisch durch Deskription" (Wengeler 2011; früher auch schon Felder 1995: 52, 59), sondern darüber hinaus auch bewertende Kritik der Verhältnisse. Leider bleibt dabei der Maßstab der Kritik - hier: "Nutzen [...] für die Allgemeinheit" und "das [...] universalistische Bedürfnis der Allgemeinheit nach vernünftigen Verhältnissen" (Feilke/Knobloch/Völzing 2007: 12) - reichlich undeutlich. Was ist die Allgemeinheit, was sind vernünftige Verhältnisse und wann ist ein Bedürfnis universalistisch? Es bleibt offen, für wen oder was Linguisten warum welche Handlungsoptionen bzw. Handlungsempfehlungen in welcher Form schaffen sollten.

Eine solche, möglichst konkrete Zweckbestimmung wissenschaftlicher Arbeit wird allerdings von nicht wenigen KollegInnen regelmäßig mit Verweis auf ihre verfassungsrechtlich begründete Freiheit von Forschung und Lehre zurückgewiesen. Diese Freiheit verbiete Fragen nach dem unmittelbarem Sinn und Zweck wissenschaftlichen Tuns schon grundsätzlich. Diese Grundhaltung hat ihre Berechtigung, sie obliegt aber in ihrem Legitimierungsversuch einem Missverständnis: denn die Wissenschaftsfreiheit nach Artikel 5 Absatz 3 GG ist vor allem ein Abwehrrecht gegen staatliche, außerwissenschaftliche Eingriffe, ein historisch begründetes 
Mahnmal gegen politische Instrumentalisierung, wie sie die Universität und die darin Arbeitenden im dritten Reich erfahren, geduldet und befördert haben. Das Bundesverfassungsgericht hat 1973 in seinem grundlegenden Urteil zur Stellung von Wissenschaftlern (BVerfGE 35, 79; 29. Mai 1973) ausgeführt, die Wissenschaftsfreiheit sei ein "Recht auf Abwehr jeder staatlichen Einwirkung auf den Prozeß der Gewinnung und Vermittlung wissenschaftlicher Erkenntnisse" (ibd.: Rn. 128). Von einem solchen, vergleichbaren unmittelbaren, staatlichen Einfluss kann heute keine Rede mehr sein, im Gegenteil: Der Rückzug der öffentlichen Hand aus der Grundfinanzierung hat die Hochschulen zwar freier, d. h. für den Staat vor allem kostengünstiger gemacht, aber letztlich der rein ökonomischen Verwertungslogik und damit dem immer häufiger direkten Einfluss der Privatwirtschaft bis in die Architektur hinein (Aldi-Hörsaal, Sparkassen-Hörsaal u. ä.) geöffnet. Eine innerwissenschaftliche Entwicklung und Diskussion von eigenen Handlungs- bzw. Bewertungsmaßstäben ist damit nicht nur erwünscht, sondern meines Erachtens auch dringend geboten.

Für einen solchen Bewertungsmaßstab oder Kompass für die linguistische Arbeit wird in verschiedenen, insbesondere diskurslinguistischen Arbeiten häufig und in der Regel im Konjunktiv auf die sog. allgemeinen Grund- und Menschenrechte verwiesen (z. B. Wengeler 2011: 4243; van Dijk 2009: 63; Jäger 2005: 68). Leider bleibt aber auch hierbei immer unklar, was denn damit jeweils gemeint sein könnte oder sollte.

Etwas mehr Text findet sich dagegen bei einer Gruppe von Sprachwissenschaftlern (vor allem Soziolinguisten), die sich seit den späten 90er Jahren für die Anerkennung sogenannter Linguistic Human Rights oder für eine "ethnolinguistische Demokratie" einsetzt (vgl. Skutnabb-Kangas et al 1995; Fishman 1995; Hamel/Valdés 1997; Skutnabb-Kangas 2012; Deklaration von 1996). Dieses Konzept der Sprach(en)rechte schließt an bisherige Menschenrechtserklärungen an und hat vor allem den Schutz von Minderheitensprachen zum Gegenstand. Teil der erklärten Rechte ist dabei etwa, die eigene Muttersprache lernen und sprechen zu dürfen und die damit verbundene Identifikation von anderen respektiert zu wissen. Von diesem Individualrecht werden dann kollektivrechtlich eine Reihe von staatlich-gesellschaftlichen Maßnahmen abgeleitet, bei denen es um einen institutionalisierten Erhalt der jeweiligen Minderheitensprache in eigenen Schulen, in den Medien und der öffentlichen Verwaltung geht.

Ingrid Piller und Kimie Takahashi (2011), die sich vor allem mit Migration und Sprache beschäftigen, und andere haben dieses Konzept - wie ich finde völlig zu Recht - als zu eng und abstrakt kritisiert.

[W] hat counts is not the existence and distribution of languages, but the availability, accessibility and distribution of specific linguistic-communicative skills such as competence in standard and literate varieties of language. Granting a member of a minority group the right to speak his or her mother tongue in the public area does not in itself empower him or her.

(Blommaert 2001: 136)

Hintergrund dieser Kritik ist die Absage an den wiederholten Versuch, Menschenrechte - welcher Art auch immer - als angeboren, universell, als allgemeingültig zu erklären. Der Menschenrechtler und heutige Emeritus Wolf-Dieter Narr hat die praktische Geschichte der großen Menschenrechtserklärungen untersucht und gezeigt, dass diese Erklärungen in der Praxis nie wirklich allgemein gültig sein sollten: 
In reality, the universal claims were rhetorical strategies to justify political shifts in power within specific historical and geographical contexts.

(Fields/Narr 1992: 3)

So erklärte die Amerikanische Unabhängigkeitserklärung 1776 - schon damals in einem deontischen Indikativ - die Gleichheit aller Menschen an unveräußerlichen Rechten, insb. Leben, Freiheit und das Streben nach Glück. Doch diese Rechte galten nur "deshalb unausgesprochen, weil westwärts selbstverständlich, 'nur' den weißen, westlichen, besitzbürgerlichen Männern" (Narr 2012: 23-24), Frauen war das Wahlrecht und Sklaven jegliche Individualrechte verwehrt. Das Motiv der Erklärung steckt bereits im Namen: es ging in erster Linie um die Interessensartikulation einer bestimmten Bevölkerungsgruppe, die sich gegenüber Großbritannien zu emanzipieren suchte, nicht um alle Menschen.

Ähnlich verhält es sich, soweit ich sehe, auch mit der Universal Declaration of Linguistic Rights (UNESCO 1996), die offenbar nicht den einzelnen Menschen, sondern vor allem Sprachgemeinschaften adressiert. Die behauptete Universalität der Sprachenrechte ist genau genommen eine nachvollziehbare und historisch legitime diskursive Strategie ethnischer Minderheiten, im Geltungsschatten anderer Menschenrechtserklärungen das eigene Existenzrecht gegenüber der Mehrheitsgesellschaft durchzusetzen.

Die Forderung nach Respekt gegenüber Minderheitensprachen sei natürlich unbenommen; als breite Grundlage für einen Orientierungsmaßstab für linguistische Gesellschaftsarbeit taugt die Erklärung meines Erachtens aber genauso wenig wie andere als universell apostrophierte Menschenrechtskataloge.

Ich möchte in Anlehnung an Narr (2012) daher ein anderes, holistisches Konzept stark machen: Menschenrechte lassen sich demnach niemals abschließend als ewige, gar angeborene oder natürliche Rechtssätze formulieren. Sie sind vielmehr versprachlichter Ausdruck von konkreten Bedürfnissen in einer konkreten gesellschaftlichen Situation und müssen als solche immer neu von den Beteiligten ausgefochten werden. Das heißt aber wiederum nicht, dass Menschenrechte völlig beliebig wären. Vielmehr zeigt ein historischer Blick auf die verschiedenen Lebensbereiche, in denen Menschen sich gegen herrschaftliche Unterdrückung erhoben haben (von der Auflehnung gegen Sklaverei bis hin zu Protesten gegenüber modernen Formen ökonomischer und sozialer Ausbeutung), dass sie zu unterschiedlichen Zeiten bestimmte Grundbedürfnisse oder - wie Narr und Fields sagen - key values gleichermaßen teil(t)en. Diese vier Grundbedürfnisse sind miteinander verschränkt und finden ihr Destillat in den Ausdrücken: Freiheit, soziale Anerkennung, kontextsensitive Gleichheit und Integrität.

First, all people long for some kind of freedom. That longing is not abstract; it is directed toward what is felt to be a lack in a given social and historical context. However slavery is defined, nobody wants or has wanted to be a slave to another.

The second key value is social recognition. If human beings are defined by their potential for selfconsciousness, this self-consciousness is dependent upon recognition from others. Despite the assertions of Hobbes and some other liberalt hinkers, human beings are not self-sufficient. No autonomy exists in that sense. Human beings need recognition because the human life is a balance between co- and self-determination. 
Third, all people want equality relative to the social context in which they live. Suppressed people may in fact accept a kind of stagnant domination built upon a rigid historical structure of inequality. But some small change in the structural precepts or some catalyst from the outside can provoke a previously stagnated, suppressed people into a struggle for social reconstruction that may only be held back, if at all, by the application of murderous coercive power (as demonstrated by La Matanza, the 1932 massacre in which approximately 30,000 mainly indigenous people were killed in a matter of weeks, and the repression and revolution in El Salvador throughout the 1980s and up to the present).

Finally, all people are striving for their own integrity. They want to believe that their lives constitute something true, something whole, something valid for themselves and for others with whom they interact.

(Fields/Narr 1992: 5-6)

In Folge ihrer historischen und situativen Kontextsensitivität kommt zu diesen Schlüsselwerten noch ein weiterer wichtiger Aspekt hinzu: Diese vier Grundbedürfnisse können nämlich nur dann annähernd erfüllt werden, wenn die Menschen über die Gestaltung ihres Zusammenlebens aktiv mitentscheiden können. Die Möglichkeit, meine individuellen Bedürfnisse zu befriedigen, findet ihre Grenzen an den Bedürfnissen und deren praktischer Umsetzbarkeit eines anderen. Kurz: Menschenrechte und Partizipation, Menschenrechte und Demokratie bedingen einander.

Partizipation setzt jedoch wiederum voraus, den eigenen Anliegen tatsächlich eine Stimme geben zu können. Ich glaube, dass damit auch historisch gesehen ein fünftes Grundbedürfnis menschlichen Zusammenlebens verbunden ist, nämlich die Fähigkeit zur kontextsensitiven Artikulation und Kommunikation, also die Fähigkeit, die eigenen Bedürfnisse verständlich zu machen. In eine ähnliche Richtung und am Problemfeld der Migration denken offenbar Piller und Takahashi (2011: 588), wenn sie schreiben:

language [is a] mediating access to human rights and we do not see that the idea of having a right to a particular language (as in the linguistic human rights concept) is tenable. [...] communication itself is a human right and being placed in a situation where one cannot communicate is a human rights concern [...].

Die Relevanz dieses Grundbedürfnisses zeigt sich bereits beim ersten Schrei des Neugeborenen, der als Ausdruck eines Bedürfnisses nach Wärme oder Nahrung und als Gesundheitsindikator medizinisch geradezu gefordert wird. Nichts erscheint uns heute gewaltsamer, als das hilflose Schreien eines Säuglings äußerlich zu unterdrücken. Aber denken wir auch an andere Situationen, in denen Menschen der Fähigkeit zum Selbstausdruck beraubt und sich dessen bewusst werden: Etwa als Fremdsprechender im Ausland bei Not, nach einem Aphasien auslösenden Schlaganfall oder gar im Stimme erpressenden Folterverhör. Wer Situationen wie die genannten erlebt hat, schildert sie als Ohnmacht, als Unfähigkeit der Selbstäußerung und damit des Selbstverlustes, als graduelles Gefühl des Ausgeliefert-Seins.

Vor dem Hintergrund dieses holistischen Menschenrechtskonzeptes sehe ich durchaus einen flexiblen, doch hinreichend bestimmten Kompass oder Wertmaßstab für linguistische Arbeiten. Die handlungsleitenden Fragen ließen sich dann wie folgt zusammenfassen: Was sind aus sprach- und kommunikationswissenschaftlicher Perspektive die Möglichkeits-bedingungen, unter denen die eben vorgestellten Grundbedürfnisse, allen voran das Bedürfnis nach kommunikativer Teilhabe an Entscheidungsprozessen, in der sozialen Praxis realisierbar sind? Wie wäre unsere Gesellschaft einzurichten, damit ihre Mitglieder im wahrsten 
Sinne des Wortes als mündige Bürgerinnen und Bürger ${ }^{3}$ eine Stimme erhalten? Wie ist demokratisches Sprechen möglich?

Ich habe auf diese Fragen an dieser Stelle keine vollständigen Antworten. Im zweiten Teil dieser Skizze möchte ich aber zumindest versuchen, einige Ansätze und damit neue Fragen bzw. Arbeitsfelder anzureißen.

\section{$3 \quad$ Aspekte demokratischen Sprechens}

Meines Erachtens gibt es zwei grundlegende, eng miteinander verschränke Domänen oder Aspekte, die aus linguistischer und kommunikationswissenschaftlicher Perspektive auf Gewährleistung der oben genannten Grundbedürfnisse hin zu prüfen wären, nämlich zum einen Lerngegenstände und Lehr-Lern-Verhältnisse im Bildungssystem, zum anderen die Medialität von Partizipationsräumen, insb. Strukturen und Zugänge des prozeduralen Rechtssystems.

\subsection{Lerngegenstände und Lehr-Lern-Verhältnisse im Bildungssystem: Die Fähigkeit zu demokratischem Sprechen lernen und erproben}

Sich selbst oder seine Bedürfnisse in unterschiedlichen Situationen gegenüber verschiedenen Adressaten angemessen zu vermitteln, ist, wie wir wissen, eine hochkomplexe Angelegenheit. Es erfordert nicht nur die Reflexion der eigenen und fremden Interessen, sondern auch Wissen über Routinen dialogischen Sprechens, den Zusammenhang von Äußerung und Äußerungskontext, über das Perspektivierungspotential der Sprache, Empathie und Antizipationsfähigkeit im Hinblick auf potentiell verletzendes oder gar gewaltsames Sprechen und vieles andere. Diese kommunikative Kompetenz muss erlernt werden - und sie wird auch erlernt, nämlich mit jeder kommunikativen Situation, in der die Interaktanten das gemeinsame Gefühl entwickeln, sich gegenseitig zu verstehen. Besser noch als das intuitive Lernen in der Praxis ist die zusätzliche Reflexion der Lernprozesse auf der Metaebene, die Fähigkeit zur Kritik im kantschen Sinne der Prämissenschau. Kommunikationsfähigkeit in diesem Sinne ist mehr als bloßer, auf Arbeitsmarktbedürfnisse zugerichteter skill. Im Sinne eines kritischen Sprach- und Diskursbewusstseins ist sie

a prerequisite for effective democratic citizenship, and should therefore be seen as an entitlement for citizens, especially children developing towards citizenship in the educational system [...]."

(Fairclough 1992/1998: 2-3; vgl. auch Fairclough 1999)

Ein solches Bewusstsein lässt sich nicht frontal eintrichtern, entsprechendes Wissen nicht kurz auswendig lernen, sondern es braucht Zeit. Mit dreizehn Jahren und acht Semestern Training stehen einem in der Regel ganz andere kommunikative Routinen der Bedürfnisartikulation und gewaltfreier Konfliktlösung zur Verfügung als nach neun oder noch weniger Jahren Schule. Wäre es aber dann nicht notwendig, finanzielle und institutionelle Rahmenbedingungen zu schaffen, die allen Bürgerinnen und Bürgern diese Basisfähigkeit garantieren?

Basisfähigkeiten beginnen mit der Alphabetisierung. Schätzungen von 2003 gehen aber davon aus, dass in Deutschland rund 4 Mio. Menschen (6,3\%) funktionale Analphabeten leben, die Mangels Beherrschung der Schriftsprache teilweise erhebliche Probleme bei der Bewältigung ihres Alltags haben (vgl. Hubertus/Nickel 2003). Die Leo-Studie der Universität Hamburg (vgl.

\footnotetext{
3 Das heißt nicht, dass damit nur Personen adressiert sind, die das formelle Bürgerrecht besitzen.
} 
Grotlüschen/Riekmann 2011) spricht 2011 von 7,5 Mio. (also mehr als $14 \%$ ) funktionalen Analphabeten unter Erwerbsfähigen, weitere 13 Mio. Menschen (25\%) zeigten mangelhafte Schreibfähigkeit.

Noch schwieriger sieht die Situation bei Migranten mit Aufenthaltstitel aus, die man seit 2005 zu sog. Integrations- und Sprachkursen zwingt, aber weder eigene staatlich finanzierte noch überhaupt ausreichend Kurse bereitstellt. 2010 fehlten laut BMBF rund 20.000 Plätze (vgl. Schüle/Topcu 2010: 3) ${ }^{4}$. Hinzu kommt, dass der individuelle Bildungshintergrund der Lernenden meist völlig ausgeblendet wird. Eine indische junge Frau mit englischem Abitur geht aber völlig anders an einen Sprachkurs heran als eine Fünfzigjährige aus dem Kongo mit Grundoder gänzlich ohne Schulabschluss, die morgens noch vor dem Kurs in der Klinik nebenan die Toiletten zu reinigen hat. Die Sprachkurse kosten übrigens zwischen 700 und 1200 Euro, Geld, das die Betroffenen häufig nicht haben. Ein staatlicher Zuschuss kann natürlich gewährt werden -, auf Antrag in deutscher Verwaltungssprache.

Wer aber in Deutschland gar als Flüchtling strandet und Asyl ersucht, dem werden im Land mit dem größten Bruttoinlandsprodukt in Europa durch Vorenthaltung einer sprachlich-kommunikativen Grundausbildung bzw. Vermittlung teilweise elementarste Grundbedürfnisse verwehrt, insbesondere rechtlicher Beistand im Asylverfahren sowie eine angemessene medizinische Versorgung. - "Asylbewerber"5 warten nach ihrer Ankunft oft viele Monate bis Jahre in Sammelunterkünften auf ihre Anhörung sowie die anschließende Entscheidung über eine (immer nur temporäre) Aufenthaltsgenehmigung des zuständigen Amtes. Das Verfahren selbst ist und bleibt ihnen meist unbekannt, genauso wie die förmlichen Schreiben der Verwaltungen. Zwar sollen Sozialarbeiter vor Ort eine kommunikative Verständigung herbeiführen, aber das scheitert in der Regel sowohl an deren Sprachkenntnisse als auch an der Arbeitskapazität (nicht selten eine Kraft auf 150 Personen). Die sog. "Anhörung", mehr ein Aushorchen, ist das Nadelöhr im deutschen Asylverfahren. Der Asylbewerber hat dort das Recht (bzw. die Auflage), die Gründe seiner Flucht darzulegen. Dies darf er in seiner Muttersprache tun, übersetzt von einem dafür amtlich bestellten Dolmetscher. Doch geht bei der Übersetzung natürlich die semantische Prosodie, das Zwischen-den-Zeilen-Stehende verloren, zumal nicht immer geeignete Dolmetscher gefunden werden oder eine Vertrauensbasis fehlt: Wie soll man gegenüber Fremden über die Erschießung des Vaters und die Vergewaltigung der Mutter berichten? Da sind Missverständnisse vorprogrammiert. Am Ende unterschreibt der Flüchtling ein Protokoll der Anhörung in für ihn unverständlicher (deutscher) Sprache, ein Dokument, das oft über Leben und (z. B. bei Rückführung von regimekritischen Afghanen in ihr Herkunftsland) Tod entscheidet.

Tödlich werden kann aber auch schon allein der Aufenthalt in Deutschland, wenn grundlegende medizinische Versorgung mangels kommunikativer Vermittlung scheitert. Was die zivilgesellschaftliche "Ehren"-Arbeit vor Ort nicht auffangen kann, bleibt unklar: Der Arzt kann keine Anamnese erstellen, schickt die Betroffenen unverrichteter Dinge fort oder verschreibt Medi-

\footnotetext{
${ }^{4}$ Derzeit steht wieder ein höherer Etat zur Verfügung, nachdem er 2006, also bereits ein Jahr nach Gesetzesinkrafttreten drastisch gekürzt worden war.

5 Das amtsdeutsche Wort Asylbewerber suggeriert, hier bewerbe sich jemand frei von allen Zwängen um einen freien "Platz" in Deutschland. Dem ist mitnichten so, wenn die Betroffenen meist mit nicht mehr als dem eigenen Hemd am Leib, teilweise von Krieg und Mord traumatisiert und durch Flucht verwirrt von einer der Polizeistreifen aufgegriffen werden.
} 
kamente auf Verdacht. Schickt der Allgemeinmediziner die Betroffenen gar weiter zum Facharzt, ist die Verwirrung groß, werden Medikamente falsch oder nicht eingenommen, droht für Manchen Lebensgefahr mangels fortgesetzter Akutmedizin.

Fälle wie diese sind keine Ausnahme, sie sind - aus eigener Erfahrung - die Regel. Sie zeigen Extremsituationen, die in gemäßigter Form aber auch für Muttersprachler im eigenen Land relevant werden. Umso drängender wäre ein systematischer Ausbau der Bildungspläne an den Stellen, wo es um kommunikative Kompetenzen geht: nicht nur im Hinblick auf streitschlichtende Kommunikationsroutinen, sondern gerade auch im Umgang mit Fachsprachen wie der medizinischen oder der juristischen. Das Verständnis der eigenen Möglichkeiten sowie kommunikativen Beschränkungen verschafft neue Handlungsoptionen, verschafft faktische (nicht nur papierne) Mündigkeit.

Sprachliche Kritikfähigkeit und kommunikative Empathie können aber schließlich nicht einfach nur Theorie bleiben. Die Bildungseinrichtungen müssen selbst so eingerichtet werden, dass sie Partizipation und Verantwortung an Entscheidungsprozessen ermöglichen und nicht verhindern. Dazu gehörte auch die Erfahrung, dass die eigene Stimme nicht nur Beiwerk für universitäre Hochglanzbroschüren ist, sondern durch verfasste Mitbestimmungsrechte auch tatsächlich Folgen für das gemeinsame Zusammenleben hat. Lehr-Lern-Verhältnisse müssten also grundständig auf gleichberechtigter Dialogizität aufbauen, von der Schule über die Universität bis zur Erwachsenen(weiter)bildung.

Sprach- und Kommunikationswissenschaftler müssen fragen, wie eine bedingungslose sprachlich-kommunikative Mindestausbildung zur Gewährleistung gesellschaftlicher Teilhabe des Einzelnen jenseits rhetorischer Phrasen ${ }^{6}$ auszusehen hätte und in diesem Sinne politisches Gehör einfordern.

\subsection{Recht und die Medialität von Partizipationsräumen}

Den wenigsten Mitbürgerinnen und Mitbürgern ist bewusst, dass unser Rechtsstaat im Grunde eine rein aus sprachlichem Handeln konstituierte und rein durch sprachliche Diskurse legitimierte Institution ist. Als solche transformiert er die direkte, unkontrollierte Gewalt sozialer Konflikte in die durch Verfahren beherrschte sprachliche Auseinandersetzung um das bessere Argument. "Der Richter zwingt den Konflikt in die Sprache und wendet ihn zu einem Kampf ums Recht" (Müller/Christensen/Sokolowski 1997: 55). Sprache sowie die tatsächliche Fähigkeit, sich im Konflikt sprachlich Gehör zu verschaffen, sind elementare Bausteine für eine auf Rechtsstaatlichkeit gegründete Demokratie:

Die Rechtfertigung erfolgt in der rechtsstaatlichen Demokratie dadurch, dass das Ausüben von Gewalt durch aufteilende, verlangsamende und kontrollierende Zwänge zur sprachlichen Offenlegung und Begründung erschwert wird. Auf dem Weg über die Normtexte [...] wird physische Gewalt in die Sprache 'gefaltet' und in dieser Form einem zur Entscheidung befugten Dritten [...] übertragen.

(Müller 1997: 50)

\footnotetext{
${ }^{6}$ Wie etwa die Phrase vom lebenslangen Lernen, die in aller Regel nicht mehr ist als die Bedienung wirtschaftlicher Privatinteressen mit einer immer weiteren Flexibilisierung von "Humankapital".
} 
Leider sind die damit verbundenen institutionellen Verfahren und die Hintergründe damit verbundener Widersprüche und Spaltungen der tatsächlichen bürgerlich-liberalen Rechtspraxis (vgl. Müller 2012: 153-320)7 den Rechtsunterworfenen - also allen BürgerInnen und Bürgern - weitestgehend unklar. Denn die juristische Textarbeit erfolgt mit einer hochgradig spezialisierten und impliziten Fachsprache (vgl. im Überblick: Jeand'Heur 1998) sowie mit besonderen Interpretationsmethoden (Müller/Christensen 2013). In dieser Arbeit spielen Gesetzestexte zwar eine notwendige, aber keine hinreichende Rolle. Die Normen stecken nicht im Gesetz, sie werden genauso wie der lebensweltliche Fall erst im Rahmen der argumentativen Verknüpfung mit vielen anderen Texten, insb. der Rechtsprechung und der juristischen (Gesetzes-) Kommentarliteratur konstituiert. So liest man in der Presse häufiger von einem neuen Computergrundrecht, das sog. "Grundrecht auf Gewährleistung der Vertraulichkeit und Integrität informationstechnischer Systeme" nach Art. 2 Abs. 1 in Verb. mit Art. 1 Abs. 1 GG, im Gesetzestext der Verfassung selbst sucht man diese Formel aber vergeblich ${ }^{8}$ (vgl. ausführlich Vogel 2012b).

All dies hat nicht nur unter Linguisten wiederholt die Forderung nach einer verständlicheren Rechtssprache laut werden lassen. Leider blieben diese Stellungnahmen häufig zu pauschal, ohne an die historisch gewordene Praxis des Rechts kritisch anzuknüpfen. Vielversprechende Ansätze, dies unmittelbar an der Quelle der Gesetzesentstehung zumindest teilweise zu ändern, werden derzeit von einem linguistischen Team unter der Leitung von Stephanie Thieme am Bundesministerium für Justiz und Verbraucherschutz (BMJV) erprobt und entwickelt (eine wissenschaftliche Begleitung der dortigen linguistischen Praxis fehlt indes bislang). Gleichwohl wird das deutsche Rechtssystem aus verschiedenen Gründen wohl nicht in Jahren und auch nicht in Jahrzehnten allgemeinverständlich werden. Umso wichtiger ist meines Erachtens, dass Sprachwissenschaftler und Juristen zusammenarbeiten, um die Vertextungsverfahren zumindest adressatenspezifisch nachvollziehbarer zu gestalten. Gesetze können nicht jeden potentiellen Konfliktfall unserer Lebenswelt vorhersehen und eindeutig regeln. Das überfordert die Sprache. Gesetze können nur Orientierungsrahmen für Aushandlungen vor Ort sein. Das wiederum setzt natürlich eine entsprechende Aufklärung in den Bildungseinrichtungen voraus, genauso wie einen öffentlichen Zugang zu allen für das Rechtssystem relevanten Texten. Die Einsicht in Sammlungen bisheriger Rechtsprechung oder gar in zentrale Gesetzeskommentare - das sind praktisch die Wörterbücher der Gesetzesbegriffes -, ist heute für Privatpersonen in der Regel nur kostenpflichtig, ab 100 Euro im Monat zu haben. Ob diese künstliche Verknappung und Kommerzialisierung nicht gegen Art. 19 Abs. 4 der Verfassung (Rechtsweggarantie)

\footnotetext{
${ }^{7}$ Im zweiten Buch (2013: 153-320) gehen Müller und Christensen der Frage nach, ob sich Gewalt im und durch Staat überhaupt in Sprache fassen lässt, und "blick[en] darauf, wie [...] der Verfassungsstaat der (europäischen) Moderne [...] mit Widerspruch und Spaltung in seinen Gesellschaften umgeht". (ibd:. 153) Der bürgerlich-liberale Rechtsstaat legitimiere sich allein durch den gesellschaftlichen Konsens, dass vor und in ihm alle Mitglieder gleich zu behandeln seien, dass Recht für alle gelte. Tatsächlich zeige das System jedoch zahlreiche strukturelle Ungleichheiten, die "wo möglich, nicht offen mit den Mitteln des Systems bearbeitet [werden]" (ibd.: 157). Es geht um "allgemein bekannte [und akzeptierte] Ungleichbehandlungen 'vor dem Gesetz'. [...] Sie werden nicht Gegenstand tiefgreifender Kontroversen, die über die Richtung der Politik entscheiden könnten. Sie beruhen auf einem der Widersprüche und sind Teil einer (Ab-)Spaltung. (Ab-)Spaltung meint rechtlich normierte, legale [und diskret vorgehende] Möglichkeiten des Verdrängens als systematische Antwort auf grundlegende Widersprüche." (ibd.: 173) Der konkrete Modus des Abspaltens vollziehe sich vor allem in Sprache, "durch Staatsmacht gestütztes Sprechen oder Verschweigen" (ibd.: 177 mit zahlreichen Beispielen).

${ }^{8}$ Dieses Grundrecht wurde 2008 vom Bundesverfassungsgericht konstituiert und argumentativ an Art. 2 Abs. 1 in Verb. mit Art. 1 Abs. 1 des Grundgesetzes gebunden (BVerfGE 120, 274).
} 
verstößt, wird noch zu prüfen sein ${ }^{9}$. Gegen das Grundbedürfnis, nicht nur Recht zu haben, sondern durch eigene Informationssuche und Interpretation auch Recht zu bekommen verstößt es meines Erachtens auf alle Fälle.

Mit dem Bewusstsein des Rechtssystems als Textstruktur stellt sich ferner auch die Frage allgemeiner nach der Medialität von Partizipationsräumen. Welche institutionalisierten Möglichkeiten der Mitsprache und der verbrieften Mitbestimmung müssen gewährleistet werden, um unterschiedliche, teilweise divergierende Bedürfnisse gleichberechtigt verhandeln zu können? Angesichts der Vielzahl und Komplexität der gesellschaftlichen Verhältnisse gibt es seit langem berechtigte Zweifel, ob es mit einem allvierjährlichen Kreuz getan ist oder ob wir nicht vielmehr Formen direkter Demokratie einführen und stärken müssten.

Gegen die direkte Demokratie gibt es eine Vielzahl an Einwänden, ich will nur zwei von ihnen herausgreifen: Ein Gegenargument ist, der Einzelne könne sich nicht immer oder auch gar nicht das Wissen aneignen, das zur adäquaten Regelung der vielschichtigen, schon für Experten kaum überschaubaren Lebenskontexte nötig wäre. Ein zweiter, mit dem ersten im Grunde zusammenhängender Einwand ist, es sei unrealistisch, dass immer alle zugleich mitreden könnten. - Nun, das mag derzeit in der Tat der Fall sein, vor allem auf Grund der Größendimensionen der zu regelnden Kontexte. Verkleinert man diese jedoch, verzichtet auf zentralistische Regelungsformen zugunsten kommunaler Zirkel, so wird die Lebenswelt sehr wohl auch für den Einzelnen intersubjektiv nachvollziehbar, lässt sich Kontextualisierung orientieren. Darüber hinaus aber ermöglichen uns neue mediale Techniken und Interaktionsräume auch neue Formen der unmittelbaren, Zeit und Raum überbrückenden gegenseitigen Abstimmung. Konzepte der E-Demokratie oder Liquid Democracy (vgl. Hernani 2011) etwa nutzen das Internet als soziale Plattform, um flexible, delegierte Voten zu ermöglichen. Aufgabe von Interaktionsforschern, Medien- und Konfliktlinguisten wäre zu prüfen, wie sich diese netzbasierten Gesprächsroutinen sowohl verfahrenstechnisch als auch im Hinblick auf bestimmte Interaktionskompetenzen optimieren ließen. Von einer darauf zielenden medienlinguistischen Ethnographie des digitalen Sprechens, der Konflikte und Verfahren ihrer Bearbeitung sind wir aber noch weit entfernt.

\section{$4 \quad$ Spracharbeit im (Spät-)Kapitalismus ${ }^{10}$}

Die Suche nach Paradigmen demokratischen Sprechens richtet den sprachwissenschaftlichen Blick auf grundlegende gesellschaftliche Praxen und Möglichkeiten ihrer partizipatorischen Öffnung. Dabei geht es nicht nur um ein Stück vom Kuchen, es geht um die ganze Bäckerei. In diesem Sinne stellt sich auch die empirische Frage nach dem Verhältnis von Sprachtheorie und Gesellschaftstheorie, die - soweit ich sehe - überwiegend (wieder ${ }^{11}$ ) ausgeblendet wird. Dies gilt insbesondere für die Frage nach dem Verhältnis von Sprache (langue), sprachlicher Praxis (parole), Sprachtheorie und (spät-)kapitalistischer Gesellschaftsordnung. Was heißt es, Sprache im Kapitalismus ${ }^{12}$ zu beschreiben? Welchen Stellenwert hat Sprache und sprachliches Handeln

\footnotetext{
${ }^{9}$ Die bisherige Praxis, nach der momentan Gerichtsurteile z. B. des BVerfG allein an ein einziges Unternehmen (Juris) zur Vermarktung übergeben werden, ist umstritten bzw. nach aktuellem Stand durch den Verwaltungsgerichtshof Baden-Württembergs als rechtswidrig erklärt worden (vgl. Urteil vom 7. Mai 2013, Akz. 10 S 281/12; abrufbar unter: http://dejure.org/2013,9140).

${ }^{10}$ Für Anregungen und Literaturhinweise danke ich Hartmut Haberland.

11 Vgl. die Kritik Bourdieus an Saussure, Chomsky und Austin (Bourdieu 2005: 47-48, 79).

12 Mit Kapitalismus bezeichne ich hier "zum einen die auf Warenproduktion, Marktwirtschaft, Investition von Kapital, Lohnarbeit und Profit beruhende Produktionsweise, zum anderen die von der Herrschaft des Kapitals
} 
in Gesellschaften, die nach globalisierten Maximen der Konsum- und Kapital-Profitmaximierung eingerichtet werden? Beschreibt etwa eine linguistische Grammatik (als Modell) nicht die Sprache, sondern vielmehr unausgesprochen Sprache im Kapitalismus? Sind Sprecher im Kapitalismus kapitalistische Sprecher und sind Sprachwissenschaften im Kapitalismus kapitalistische Sprachwissenschaften? Unterstellt man, Sprache als langue besitze keine eigene ontische Qualität, sondern sei ein heuristisches Modell zur idealtypischen Beschreibung wiederkehrender sozialsemiotischer Handlungen, Abbilder gesellschaftlicher Routinen und Rituale, dann wäre man leicht dazu geneigt, Fragen wie diese zu bejahen. Doch was folgte aus solchen Aussagen bzw. Hypothesen? Kann es eine linguistische Kritik (sowohl im Kant'schen als auch im bewertenden Sinne) kapitalistischer Gesellschaftsordnung geben, die über eine überwiegend abstrakte Ideologieforschung oder Sprachenpolitik ${ }^{13}$ hinausgeht?

Diese Frage ist nicht leicht zu beantworten, vereinfachende Kurz- und Zirkelschlüsse ${ }^{14}$ liegen auf der Hand. So wurde etwa die These vertreten, Sprache fungiere - analog zu den ökonomischen Lebensbedingungen - ebenso als Arbeit und Markt (vgl. Rossi-Landi 1968/1974). Sprachliche Ausdrücke werden zwar in der Tat "produziert", d. h. Gedanken durch energetischen Aufwand als lautliche oder graphische Formen realisiert (Arbeit); Sprache als langue ist aber keine Handelsware, pauschal vergleichbar mit veräußerbaren Produkten als Teil eines nach Profitmaximierung und -akkumulierung strebenden Verarbeitungsprozesses.

It can be agreed that words and expressions, as products of human linguistic labor, possess value (they are the product of labor) and consumer value (they are actually called upon to satisfy different human needs: expressing, designating, informing, characterizing, conveying a request or command etc.). It is true that they are created in order to be passed on to another, and to be 'consumed', i.e., to be understood. It is true that linguistic units are dead if not used, so are machines and implements without human labor. But not every product of human labor is a commodity. If a commodity is defined as a product satisfying human needs and destined for sale, then only the first part of this definition applies to linguistic units.

(Gak 1975: 23-24)

Deutlich differenzierter untersucht Bourdieu (2005) vor der Folie seiner Theorie der Praxis die Komplexität sprachlicher Interaktion als "Ökonomie des symbolischen Tauschs". Sprachen (Varietäten, Stile) erscheinen hier zwar auch als umkämpfte, miteinander konkurrierende Angebots-"Produkt[e]", als "Güter, die einen Preis bekommen sollen, und Werte, die Kredit verschaffen können (unterschiedlich je nach dem Markt, auf dem sie angelegt sind)" (ibd: 84). Sie gewinnen ihren Wert jedoch nur symbolisch in der Repräsentation und Konstitution kulturellen Kapitals, also als inkorporierte Indices für soziale Herkunft, Bildungshintergrund usw. Wer seine sprachlichen Ressourcen möglichst effektiv einsetzen kann, d. h. insbesondere auf gesellschaftlichen Handlungsfeldern, die mit der eigenen Sozialisation kohärent sind und damit sicheres Selbstbewusstsein stiften, kann daraus "Distinktionsprofit" (ibd.: 61) schlagen und andere Kapitalformen (Anerkennung, Zugang zu Bildung oder lukrativen Jobs etc.) erschließen.

\footnotetext{
bedingten sozialen, politischen, rechtlichen und kulturellen Verhältnisse als Gesellschaftsordnung" (HKWM 7/I, 2008: Sp. 238). Für eine differenzierte Beschreibung spätkapitalistischer Entwicklung aus der Perspektive der Mehrsprachigkeitsforschung vgl. Heller/Duchêne 2012.

13 Vgl. zuletzt etwa Duchêne/Heller 2012 oder die Beiträge der Zeitschrift Language \& Capitalism.

14 Wie etwa häufig der Kritischen Diskursanalyse (teilweise auch zu Recht) vorgeworfen wurde: etwa dass ein Diskurs per se als rassistisch anzusehen sei, weil in einer rassistischen Gesellschaft verortet $u$. ä.
} 
Die Struktur des Raums der Sprachstile reproduziert [...] die Struktur der objektiven Unterschiede der Existenzbedingungen, und zwar vermittelt über die Struktur des sprachlichen Feldes als eines Systems sprachlicher Machtverhältnisse im eigentlichen Sinne, die auf der ungleichen Verteilung des Sprachkapitals beruhen (oder, wenn man so will, auf der ungleichen Verteilung der Chancen, objektivierte sprachliche Ressourcen zu inkorporieren).

(ibd.: 63)

Sprachkompetenz ist damit keine abstrakte Kategorie idealer Sprecherhörer (wie bei Chomsky), sondern als Teil des sprachlichen Habitus ganz praktischer Ausdruck und reproduzierender Faktor sozialer Unterschiede und Hierarchien (vgl. ibd: 60).

in jeder Interaktion (und damit bei jedem Diskurs) ist über die verwendeten Sprachen, über ihre Sprecher und über die sozialen Gruppen - definiert über den Besitz der entsprechenden Sprachkompetenz - die ganze Sozialstruktur präsent.

(ibd. 74)

Bourdieu vermittelt mit diesem Ansatz zwischen einerseits Sprachen in ihrer Rolle als soziale Distinktionsmedien in gesellschaftlichen, Markt-orientierten Makrosystemen und andererseits sprachlicher Performanz in der konkreten sozialen Interaktion auf der Mikroebene.

In eben diesem Sinne ließe sich auch weiter fragen, inwiefern sich originär kapitalistische Konzepte oder Maximen in den filigraneren Strukturen wiederfinden, also auf der Ebene der Lexik, Syntax, Grammatik, der Gesprächsroutinen? Doch diese Frage droht bereits an der Operationalisierung zu scheitern: Eine kapitalistische, oligarchische, diktatorische usw. Lexik oder Grammatik müsste ja gegenüber nichtkapitalistischer usw. Lexik oder Grammatik abgegrenzt werden. Auch wäre zu differenzieren zwischen verschiedenen Domänen (z. B. formelle vs. informelle Sphären) und Herrschaftsebenen (z. B. Sprache von Subalternen vs. Sprache dominierender Herrschaftsschichten). Schließlich ist Sprache ja nicht nur passives Medium, quasi hilfloses Auffangbecken kapitalistischer Indoktrination, sondern auch Medium der Resistance, des subversiven Widerstands.

Völlig aussichtslos scheint das Unternehmen dennoch nicht zu sein: Im Bereich der Lexik ließe sich etwa auf Basis großer Korpora und mithilfe computerlinguistischer Methoden diachron prüfen, ob Spezial- und Fachlexika und die damit verbundenen semantischen Hüllräume (d. h. sprachlichen Gebrauchskontexte) - etwa aus spezifischen Ökonomiediskursen - sich sukzessive auch auf andere Lexikbereiche bzw. Wissensdomänen des gesellschaftlichen Lebens wie Bildungs- und Gesundheitsdiskurse verbreiten und diese framen. Indizien (nicht Belege!15) etwa für eine Expansion neoliberaler Konzepte lassen sich anhand einfacher google-ngramsAbfragen illustrieren: ${ }^{16}$

\footnotetext{
${ }^{15}$ Die Interpretation von Wortfrequenzen insb. in undokumentierten Großkorpora (wie sie bei Google vorliegen) birgt zahlreiche Probleme.

16 http://goo.gl/1EJ6FF, letzter Zugriff am 29.12.2014).
} 


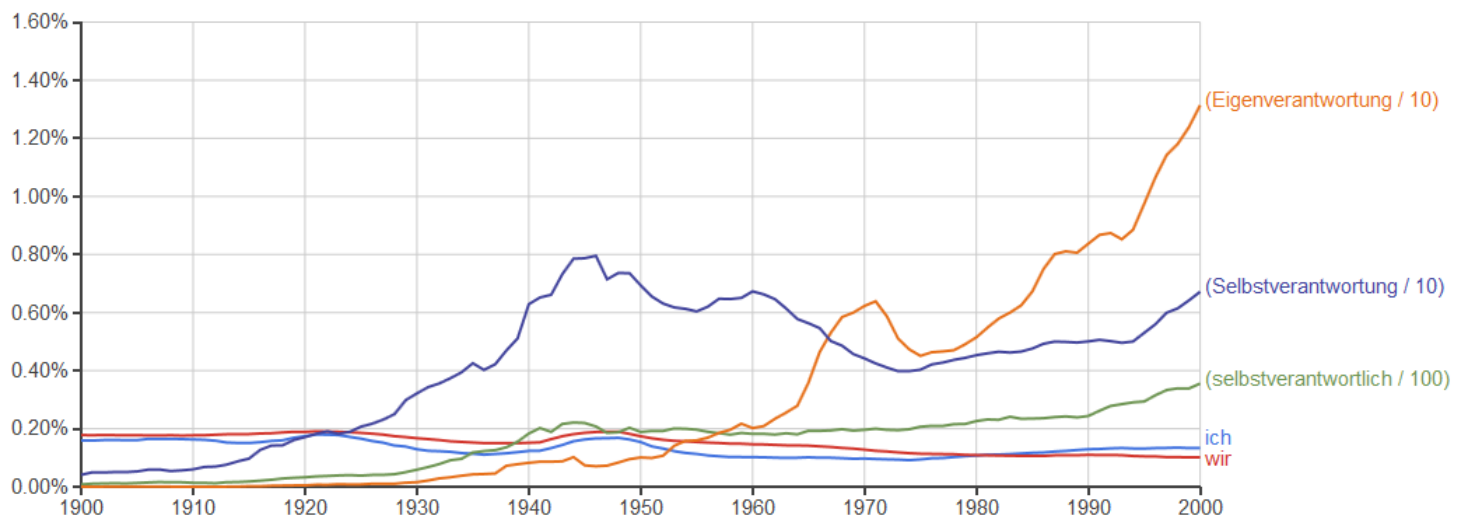

Abb. 1: Diachroner Verlauf der relativen Frequenzen von ausgewählten Ausdrücken im Kontext von Google-ngrams als Indikatoren für eine Expansion neoliberaler Konzepte.

Auch im Bereich der Gesprächsroutinen lassen sich Einflüsse kapitalistischer Verwertungslogik nachzeichnen: So wird Sprechen in manchen Bereichen zunehmend dehumanisiert, $d . h$. mit Menschen als "Spracharbeiter" automatisiert. Boutet (2012) beschreibt etwa eine zunehmende Standardisierung und Taylorisierung der Arbeitsprozesse in Call-Center als quasi "Sprachfabriken" und resümiert:

More than one hundred years after the invention of the rationalization of manual labor and piecework, the worldwide economy of information and communications has made it possible to rationalize the processes of articulation. The logics of standardization, of voice, of discourse and of languages, if pushed to their limits, would lead to the disappearance of employees in this sector: they would be replaced by vocal robots, as is already the case in many companies where guiding the client is done by a voice server.

(Boutet 2012: 223)

Lassen sich ähnliche, ansonsten als "natürlich" apostrophierte Veränderungen in der BehördenBürger-Kommunikation feststellen und wenn ja, wie sind diese zu bewerten (vom Amt zur Agentur, vom Bürger zum Klienten, vom Bürgeranliegen zur behördlichen Dienstleistung, von Partizipation zu marktkonformer Demokratie usw.)?

Schließlich wäre zu fragen, welche Rolle Linguistik und Linguisten selbst in kapitalistischen Gesellschaftsordnungen einnehmen. Welche systemischen Erwartungen werden implizit wie explizit an Sprach- und Kommunikationswissenschaft herangetragen und - zumal in Zeiten knapper öffentlicher Kassen und der Abhängigkeit von Drittmitteln - möglicherweise in Theorie und Empirie auch bereits bedient?

\section{$5 \quad$ Fazit}

Der vorliegende Beitrag versteht sich als Plädoyer für eine "Linguistik als Kampfsport", eine Linguistik, die am Maßstab eines holistischen Menschenrechtskonzeptes versucht, in die gesellschaftliche Praxis einzugreifen und Stolpersteine auf die Straßen des kommunikativen Alltags zu setzen. Es geht darum, im semantischen Kampf um gesellschaftliche Vorrangstellungen und Asymmetrien für Waffengleichheit unter den beteiligten Sprecherinnen und Sprechern zu sorgen. Hierzu gehört insbesondere die gesellschaftliche Gewährleistung der Fähigkeit zur kontextsensitiven Bedürfnisartikulation des Einzelnen gegenüber seinen Mitmenschen ineins mit der Möglichkeit zur Partizipation an gesellschaftlichen Entscheidungsvorgängen. Sprach- und 
Kommunikationswissenschaftlern kommt dabei die Aufgabe zu, die theoretischen wie praktischen Möglichkeitsbedingungen für diese Maximen in der real existierenden Marktwirtschaft, also unter den Vorzeichen neoliberaler, kapitalistischer Gesellschaftsordnung zu untersuchen und Schlussfolgerungen auch aktiv in die Arenen politischer Aushandlung zu tragen.

Das hier Skizzierte fasst bislang weit mehr Fragen als Antworten und vieles wird man nicht alleine, sondern nur im Verbund mit Kolleginnen und Kollegen anderer Fachdisziplinen lösen können. Auch wäre dabei wohl dringend eine Arbeitsform zu finden, die sich zumindest ein Stück weit für die nicht-akademische Welt öffnet.

"[E]ines ist jedenfalls sicher: nichts ist weniger unschuldig, als den Dingen einfach ihren Lauf zu lassen." (Bourdieu 1997: 826). In diesem Sinne ist das in der Linguistik ebenfalls weithin vernachlässigte Thema "Sprache und Demokratie" Gegenstand einer interdisziplinären Tagung vom 02.-03. Oktober 2014 unter Leitung von Clemens Knobloch und Friedemann Vogel. Ziel der Tagung ist, inner- und außerhalb der wissenschaftlichen Community für das spannungsvolle Verhältnis der beiden Phänomenbereiche zu sensibilisieren, aktuelle Konfliktherde und Problemstellungen auf sprach-gesellschaftlicher Makro- wie Mikroebene zu benennen, ihre historische Genese zu analysieren und Ansätze für lösungsorientierte, weiterführende und möglicherweise effektiver organisierte Forschungsprogramme zu skizzieren. Nähere Informationen zur Tagung finden sich unter: www.sud.friedemann-vogel.de.

\section{Literatur}

Blommaert, Jan (2001): "The Asmara Declaration as a Sociolinguistic Problem. Reflections on Scholarship and Linguistic Rights. Journal of Sociolinguistics 5:§ 131-155.

Bourdieu, Pierre (1997): Das Elend der Welt. Konstanz: UVK Universitätsverlag.

Bourdieu, Pierre/Beister, Hella (2005): Was heißt sprechen? Zur Ökonomie des sprachlichen Tausches. 2. Aufl. Wien: Braumüller.

Bourdieu, Pierre; Pialoux, Cordula; Schwibs, Bernd (2009): Entwurf einer Theorie der Praxis. Auf der ethnologischen Grundlage der kabylischen Gesellschaft. 2. Aufl. Frankfurt am Main: Suhrkamp (Suhrkamp-Taschenbuch Wissenschaft, 291).

Boutet, Josiane (2012): "Language Workers. Emblemic Figures of Late Capitalism". In: Duchêne, Alexandre/Heller, Monica (eds.): Language in Late Capitalism. Pride and Profit. New York, Routledge: 207-229. (= Routledge Critical Studies in Multilingualism 1).

Carles, Pierre/Bourdieu, Pierre (2009): Soziologie ist ein Kampfsport. Pierre Bourdieu im Porträt. Frankfurt a. M./Berlin: Suhrkamp/Absolut Medien. DVD-Video.

Coulmas, Florian (2009): "Democracy and the crisis of normative linguistics". In: Coulmas, Florian (ed.): Language adaptation. Cambridge, Cambridge University Press: 177-193.

Dijk, Teun A. van (2009): "Critical Discourse Studies. A Sociocognitive Approach". In: Ruth Wodak, Ruth/Meyer, Michael (eds.): Methods for Critical Discourse Analysis. London, Sage Publications: 62-85.

Duchêne, Alexandre; Heller, Monica (eds.) (2012): Language in Late Capitalism. Pride and Profit. New York: Routledge. (= Routledge Critical Studies in Multilingualism 1).

Fairclough, Norman (ed.) (1992/1998): Critical language awareness. 3. Aufl. London: Longman. (= Real language series).

Fairclough, Norman (1999): "Global Capitalism and Critical Awareness of Language". Language Awareness 8/2: 71-83. 
Feilke, Helmuth/Knobloch, Clemens/Völzing, Paul-Ludwig (2007): "Was heißt "linguistische Aufklärung"? Sprachauffassungen zwischen Systemvertrauen und Benutzerfürsorge". In: Feilke, Helmuth/Knobloch, Clemens/Völzing, Paul-Ludwig (eds.): Was heißt linguistische Aufklärung? Heidelberg, Synchron: 9-20.

Felder, Ekkehard (1995): Kognitive Muster der politischen Sprache. Eine linguistische Untersuchung zur Korrelation zwischen sprachlich gefaßter Wirklichkeit und Denkmustern am Beispiel der Reden von Theodor Heuss und Konrad Adenauer. Frankfurt a. M. etc.: Lang.

Fields, A. Belden/Narr, Wolff-Dieter (1992): "Human Rights as a Holistic Concept". Human Rights Quarterly 14/1: 1-20.

Fishman, Joshua A. (1995): "On the Limits of Ethnolinguistic Democracy". In: Skutnabb-Kangas, Tove/Phillipson, Robert/Rannut, Mart (eds.): Linguistic Human Rights. Overcoming Linguistic Discrimination. Berlin, Mouton de Gruyter: 49-62. (=Contributions to the Sociology of Language 67).

Gak, V. G. (1975): "Language, Implement and Commodity". In: Linguistics 13/143: 17-32.

Grotlüschen, Anke/Riekmann, Wiebke (2011): leo. Level-One Studie. Literalität von Erwachsenen auf den unteren Kompetenzniveaus. Hamburg. Presseheft. http://blogs.epb.uni-hamburg.de/leo/files/2011/12/leo-Presseheft_15_12_2011.pdf, letzter Zugriff am 20.01.2014.

Hamel, Rainer E./Valdés, Guadalupe (eds.) (1997): Linguistic Human Rights from a Sociolinguistic Perspective: Berlin/New York: Mouton de Gruyter. (= International Journal of the Sociology of Language).

Haug, Wolfgang Fritz; Haug, Frigga; Jehle, Peter (seit 1997): Historisch-kritisches Wörterbuch des Marxismus. Berlin: Argument-Verl.

Heller, Monica/Duchêne, Alexandre (2012): "Pride and Profit. Changing Discourses of Language, Capital and Nation-State". In: Duchêne, Alexandre/Heller, Monica (eds.): Language in Late Capitalism. Pride and Profit. New York, Routledge: 1-21. (= Routledge Critical Studies in Multilingualism 1).

Hernani, Marques (2011): "Liquid Democracy - Zwischen repräsentativer und direkter Demokratie". In: Sociology in Switzerland (ed.): Democracy in Politics and Social Life. Zürich. http://socio.ch/demo/t_marques.pdf, letzter Zugriff am 20.01.2014.

Hubertus, Peter/Nickel, Sven (2003): "Alphabetisierung von Erwachsenen". In: Ursula Bredel, Ursula/Günther, Hartmut/Klotz, Peter/Ossner, Jakob/Siebert-Otto, Gesa (eds.): Didaktik der deutschen Sprache - Ein Handbuch. 2 Bände. Paderborn etc., Schöningh: 719-728. (= Didaktik der deutschen Sprache 2).

Jäger, Siegfried (2005): "Diskurs als "Fluß von Wissen durch die Zeit". Ein transdisziplinäres politisches Konzept". Aptum. Zeitschrift für Sprachkritik und Sprachkultur 1/1: 52-72.

Jeand'Heur, Bernd (1998): "Die neuere Fachsprache der juristischen Wissenschaft seit der Mitte des 19. Jahrhunderts unter besonderer Berücksichtigung von Verfassungsrecht und Rechtsmethodik". In: Hoffmann, Lothar et al. (eds.): Fachsprachen. Ein internationales Handbuch der Fachsprachenforschung und Terminologiewissenschaft. Berlin, de Gruyter: 1286-1295. (= Handbücher zur Sprach- und Kommunikationswissenschaft 1).

Linke, Angelika et al. (2001): Studienbuch Linguistik. Ergänzt um ein Kapitel "Phonetik und Phonologie" von Urs Willi. 4. Aufl. Tübingen: Niemeyer. (= Reihe germanistische Linguistik Studienbuch 121). 
Müller, Friedrich (1997): "Demokratie, Rechtsarbeit, Volksgemurmel". In: Friedrich Müller, Friedrich/Christensen, Ralph (eds.): Methodik, Theorie, Linguistik des Rechts. Neue Aufsätze (1995 - 1997). Berlin, Duncker \& Humblot: 20-35. (= Schriften zur Rechtstheorie 181).

Müller, Friedrich (2012): Syntagma. Verfasstes Recht, verfasste Gesellschaft, verfasste Sprache im Horizont von Zeit. Berlin: Duncker \& Humblot.

Müller, Friedrich/Christensen, Ralph (2013): Juristische Methodik. Grundlegung für die Arbeitsmethoden der Rechtspraxis. 11., auf neuestem Stand bearb. und erw. Aufl. Berlin: Duncker \& Humblot.

Müller, Friedrich/Christensen, Ralph/Sokolowski, Michael (1997): Rechtstext und Textarbeit. Berlin: Duncker \& Humblot. (= Schriften zur Rechtstheorie 179).

Narr, Wolf-Dieter (2012): Trotzdem: Menschenrechte! Versuch, uns und anderen nach nationalsozialistischer Herrschaft Menschenrechte zu erklären. Unter Mitarbeit von Dirk Vogelskamp. Köln: Komitee für Grundrechte und Demokratie.

Piller, Ingrid/Takahashi, Kimie (2011): "Language, Migration and Human Rights". In: Ruth Wodak, Ruth/Johnstone, Barbara/Kerswill, Paul (eds.): The Sage Handbook of Sociolinguistics. London, Sage: 583-597.

Richardson, John E./Roderrick, Ian (eds.): Language \& Capitalism. http://www.languageand capitalism.info/, letzter Zugriff am 01.12.2014.

Rossi-Landi, Ferruccio (1968/1974): Sprache als Arbeit und als Markt. Aus dem Italienischen von Burkhart Kroeber. 2. Aufl. München: Hanser. (= Reihe Hanser 105)

Schüle, Christian/Topcu, Özlem (2010): "Die Deutschstunde. Integrationskurse für Migranten". Die Zeit 21.06.2010. http://www.zeit.de/2010/25/DOS-Deutschstunde, letzter Zugriff am 01.12.2014.

Skutnabb-Kangas, Tove (2012): "Linguistic Human Rights". In: Tiersma, Peter. M./Solan, Lawrence (eds.): The Oxford Handbook of Language and Law. Oxford, Oxford University Press: 235-247. (= Oxford Handbooks in Linguistics X).

Skutnabb-Kangas, Tove/Phillipson, Robert/Rannut, Mart (eds.) (1995): Linguistic human rights. Overcoming linguistic discrimination. Berlin: Mouton de Gruyter, (= Contributions to the sociology of language 67).

UNESCO (ed.) (1996): Universal Declaration on Linguistic Rights. World Conference on Linguistic Rights. http://www.unesco.org/cpp/uk/declarations/linguistic.pdf, letzter Zugriff am 17.01.2014.

Vogel, Friedemann (2012a): "Linguistische Diskursanalyse als engagierte Wissenschaft?! Ein Plädoyer für eine "Theorie der Praxis als Praxis"'". In: Meinhof, Ulrike H./Reisigl, Martin/Warnke, Ingo H. (eds.): Diskurslinguistik im Spannungsfeld von Deskription und Kritik. Berlin, Akademie Verlag: 279-298. (=Diskursmuster - Discourse Patterns 1).

Vogel, Friedemann (2012b): Linguistik rechtlicher Normgenese. Theorie der Rechtsnormdiskursivität am Beispiel der Online-Durchsuchung. Berlin [u.a.]: De Gruyter (Sprache und Wissen, 9).

Vogel, Friedemann (ed.): Sprache und Demokratie - Language and Democracy. http://www.sud.friedemann-vogel.de/, letzter Zugriff am 01.12.2014.

Weber, Max (1919/2002): "Wissenschaft als Beruf". In: Weber, Max: Schriften 1894-1922. Stuttgart, Kröner: 474-511. 
Wengeler, Martin (2011): "Linguistische Diskursanalysen - deskriptiv, kritisch oder kritisch durch Deskription?" In: Schiewe, Jürgen (ed.): Sprachkritik und Sprachkultur. Konzepte und Impulse für Wissenschaft und Öffentlichkeit. Bremen, Hempen: 35-48.

Wimmer, Rainer (1982/1988): "Überlegungen zu den Aufgaben und Methoden einer linguistisch begründeten Sprachkritik". In: Heringer, Hans J. (ed.): Holzfeuer im hölzernen Ofen. Aufsätze zur politischen Sprachkritik. 2. Aufl. Tübingen, Narr: 290-316.

Wittgenstein, Ludwig (193/2003): Tractatus logico-philosophicus. Tagebücher 1914-1916. Philosophische Untersuchungen. Hg. v. Joachim Schulte. 15. Aufl. Frankfurt a. M.: Suhrkamp. (Suhrkamp-Taschenbuch Wissenschaft 501). 